\title{
Novel siRNA Delivery System Using a Ternary Polymer Complex with Strong Silencing Effect and No Cytotoxicity
}

\author{
Yukinobu Kodama, Yumi Shiokawa, Tadahiro Nakamura, Tomoaki Kurosaki, Keisei Aki, \\ Hiroo Nakagawa, Takahiro Muro, Takashi Kitahara, Norihide Higuchi, and Hitoshi Sasaki* \\ Department of Hospital Pharmacy, Nagasaki University Hospital; 1-7-1 Sakamoto, Nagasaki 852-8501, Japan. \\ Received January 20, 2014; accepted June 8, 2014
}

We developed a novel small interfering RNA (siRNA) delivery system using a ternary complex with polyethyleneimine (PEI) and $\gamma$-polyglutamic acid ( $\gamma$-PGA), which showed silencing effect and no cytotoxicity. The binary complexes of siRNA with PEI were approximately $73-102 \mathrm{~nm}$ in particle size and $45-52 \mathrm{mV}$ in $\zeta$-potential. The silencing effect of siRNA/PEI complexes increased with an increase of PEI, and siRNA/PEI complexes with a charge ratio greater than 16 showed significant luciferase knockdown in a mouse colon carcinoma cell line regularly expressing luciferase (Colon26/Luc cells). However, strong cytotoxicity and blood agglutination were observed in the siRNA/Lipofectamine complex and siRNA/PEI16 complex. Recharging cationic complexes with an anionic compound was reported to be a promising method for overcoming these toxicities. We therefore prepared ternary complexes of siRNA with PEI (charge ratio 16) by the addition of $\gamma$-PGA to reduce cytotoxicity and deliver siRNA. As expected, the cytotoxicity of the ternary complexes decreased with an increase of $\gamma$-PGA content, which decreased the $\zeta$-potential of the complexes. A strong silencing effect comparable to siRNA/Lipofectamine complex was discovered in ternary complexes including $\gamma$-PGA with an anionic surface charge. The high incorporation of ternary complexes into Colon 26/Luc cells was confirmed with fluorescence microcopy. Having achieved knockdown of an exogenously transfected gene, the ability of the complex to mediate knockdown of an endogenous housekeeping gene, glyceraldehyde 3-phosphate dehydrogenase (GAPDH), was assessed in B16-F10 cells. The ternary complex (siRNA/PEI16/ $\gamma$-PGA12 complex) exhibited a significant GAPDH knockdown effect. Thus, we developed a useful siRNA delivery system.

Key words gene delivery; small interfering RNA; $\gamma$-polyglutamic acid; ternary complex

Research into RNA interference (RNAi) has advanced rapidly since it was first discovered in 1998. ${ }^{1)}$ RNAi is a natural mechanism conserved in plant and mammalian cells that results in specific silencing of a target gene by short sequences of RNA, known as small interfering RNA (siRNA). ${ }^{2)}$ siRNA is a double-stranded RNA (dsRNA) composed of 21-23 nucleotides, and its ability to induce sequence-specific RNAimediated down-regulation of complementary mRNA has been demonstrated, resulting in knockdown of a target gene protein at the post-transcriptional level. ${ }^{3-5)}$ Numerous studies have used siRNAs as potential therapeutic agents for treating various diseases, including cancer and other diseases as a result of genetic disorders or viral infection. ${ }^{6-8)}$

Despite the immense therapeutic potential of this technology, effective systemic and intracellular delivery of siRNA is quite limited because of its rapid degradation by plasma nucleases and poor penetration of the plasma membrane of target cells. ${ }^{9,10)}$ Therefore, the establishment of an siRNA delivery system that enables prolonged activity without degradation by nucleases and efficient cytosolic delivery of siRNA into target cells is indispensable for the development of siRNA pharmaceutics. Both cationic and neutral lipoplexes have exhibited good efficacy with different toxicity and protein adsorption. ${ }^{11-15)}$ Previously, we also reported that lipoplexes of siRNA constructed with trimethyl[2,3(dioleoxy)-prolyl]ammonium chloride and cholesteryl $3 \beta-N$ (dimethylaminoethyl)carbamate hydrochloride showed a significant silencing effect in a mouse colon carcinoma cell line expressing luciferase regularly. ${ }^{16)}$

The authors declare no conflict of interest.
On the other hand, cationic polymers are one of the most popular non-viral gene vectors due to their excellent ability to compact anionic genes, synthetic controllability, better flexibility achieved simply by varying the chemical composition, molecular weight (MW), and architecture (linear, randomly branched, dendrimer, block, and graft copolymer). ${ }^{17)}$ Cationic polymers, such as chitosan and other natural polymers, polyethyleneimine (PEI), dendrimers, and other synthetic polymers have also shown promise. In particular, $\mathrm{PEI}^{18)}$ showed high gene expression in vitro and in vivo because it strongly interacts with the cell surface, and is well taken up by cells due to its strong cationic surface. Moreover, PEI achieves efficient escape from endosomes to the cytoplasm via the $\mathrm{pH}$ buffering effect and the gene released from the vector in the cytoplasm expresses the protein in the nucleus.

Nevertheless, the cationic surface of complexes with cationic polymers induced cytotoxicity and blood agglutination caused by its strong interaction with anionic surface of cells and erythrocytes. ${ }^{19-21)}$ Agglutination of the complexes often led to its rapid elimination and adverse effects, such as embolism and inflammatory reactions. ${ }^{22-24)}$ Therefore, there remains a need for the development of alternative less toxic vectors for siRNA delivery. Recharging cationic complexes with an anionic compound was reported to be a promising method for overcoming these toxicities. ${ }^{25,26)}$ In our previous study, we discovered anionic polymers such as $\gamma$-polyglutamic acid $(\gamma$-PGA $),{ }^{27)}$ chondroitin sulfate, ${ }^{28)}$ hyaluronic acid, ${ }^{28)}$ which decreased the toxicities of cationic complexes while maintaining high transgene efficiency. Among them, the addition of $\gamma$-PGA showed the highest gene expression. However, there is no certainty that ternary complex containing siRNA 
is effective as well as that containing plasmid DNA (pDNA) because the action site of pDNA differs from that of siRNA. Also, in the preliminary study, we could not prepare the ternary complex containing siRNA under the same condition which prepared the ternary complex containing pDNA.

In the present study, we developed a modified polyplex of siRNA with PEI by the addition of $\gamma$-PGA and investigated the efficacy and safety of this ternary complex as a novel siRNA delivery system.

\section{MATERIALS AND METHODS}

Chemicals and Reagents Bovine serum albumin (BSA) and PEI (branched form, average MW of 25000) were obtained from Sigma-Aldrich Co., LLC (St. Louis, MO, U.S.A.). The $\gamma$-PGA (average molecular weight; 55000) was provided by Yakult Pharmaceutical Industry Co., Ltd. (Tokyo, Japan). Lipofectamine RNAiMAX, Alexa Fluor 555-labeled siRNA (BLOCK-iT Alexa Fluor Red Fluorescent Oligo), and primers for glyceraldehyde 3-phosphate dehydrogenase (GAPDH) and $\beta$-actin (GAPDH: 5'-CTCACTCAA GAT TGTCAGCAA TG-3' (forward) and 5'-GGCAGTGAT GGCATG GAC TGT-3' (reverse), $\beta$-actin: 5 '-GTTCTACAA ATGTGGCTGAGGACT T-3' (forward) and 5'-TTGGGA GGGTGA GGGACT T-3' (reverse)) were purchased from Invitrogen (Carlsbad, CA, U.S.A.). Firefly luciferase siRNA (sense: 5'-CUUACGCUGAGUACUUCGAdTdT-3', antisense: 5'-UCGAAGUACUCAGCGUAAGdTdT-3') and scramble siRNA (sense: 5'-CUUACGCUGUCAUGAUCGAdTdT-3,' antisense: 5'-UCGAUCAUGACAGCGUAAGdTdT-3') were obtained from GeneDesign, Inc. (Osaka, Japan). The mouse GAPDH siRNA (sense: 5'-CAA GAG AGG CCCUAUCCCAdTdT-3', antisense: 5'-UGGGAUAGGGCCUCUCUUGdTdT-3') was purchased from Sigma-Aldrich Japan (Tokyo, Japan). Fetal bovine albumin (FBS) was obtained from Biological Industries Ltd. (Kibbutz Beit Haemek, Israel). For the cell culture, RPMI 1640, OptiMEM I and antibiotics (penicillin $100 \mathrm{U} / \mathrm{mL}$ and streptomycin $100 \mu \mathrm{g} / \mathrm{mL}$ ) were purchased from GIBCO BRL (Grand Island, NY, U.S.A.). The antibiotic G418 solution was obtained from Roche Diagnostics (Indianapolis, IN, U.S.A.). The 2-(4-iodophenyl)-3-(4-nitrophe-nyl)-2H-tetrazolium, monosodium salt (WST-1) and 1-methoxy-5-methylphenazinium methylsulfate (1-methoxy PMS) were purchased from Dojindo Laboratories (Kumamoto, Japan). FluoresceinisothiocyanatePEI (FITC-PEI) was prepared in our laboratory. Briefly, PEI and FITC were dissolved in dimethyl sulfoxide (DMSO) and stirred overnight at room temperature in the dark. FITC-PEI was purified by gel filtration. Almost $0.73 \%$ PEI nitrogen was labeled with FITC. All other chemicals were of reagent grade.

Preparation of Complex In this study, we constructed complexes at a theoretical charge ratio of phosphate of siRNA, nitrogen of PEI, and carboxylate of $\gamma$-PGA.

To prepare binary complexes, an appropriate amount of stock PEI solution was mixed with siRNA dissolved in diethylpyrocarbonate (DEPC)-treated water (Invitrogen) (1 mg/ $\mathrm{mL}$ ) by pipetting thoroughly at charge ratios $4,8,12,16$, 20, and 24 of PEI to siRNA (siRNA/PEI4, 8, 12, 16, 20, and 24 complexes), and left for $30 \mathrm{~min}$ at room temperature. To prepare ternary complexes, $\gamma$-PGA solution was added to siRNA/PEI16 complex at charge ratios of $4,8,12,16$, and 20 of $\gamma$-PGA to siRNA (siRNA/PEI16/ $\gamma$-PGA4, 8, 12, 16, and 20 complexes) and left for another $30 \mathrm{~min}$ at room temperature.

siRNA/Lipofectamine complex was prepared as a product protocol and used as a positive control.

Physicochemical Properties of Complex The particle size and $\zeta$-potential of complexes were measured with Zetasizer Nano ZS (Malvern Instruments Ltd., Malvern, U.K.). The number-fractioned mean diameter is shown.

To determine the complex formation, $20 \mu \mathrm{L}$ aliquots of complex solution containing $1 \mu \mathrm{g}$ siRNA were mixed with $4 \mu \mathrm{L}$ loading buffer $(30 \%$ glycerol and $0.2 \%$ bromophenol blue) and loaded on a $2 \%$ agarose gel. Electrophoresis (iMupid J; Cosmo Bio Co., Ltd., Tokyo, Japan) was carried out at $100 \mathrm{~V}$ in running buffer solution $(40 \mathrm{~mm}$ Tris $-\mathrm{HCl}, 40 \mathrm{~mm}$ acetic acid, and $1 \mathrm{~mm}$ ethylenediaminetetraacetic acid (EDTA)) for $20 \mathrm{~min}$. The retardation of siRNA was visualized with ethidium bromide staining using a Gel Doc EZ System (BioRad Laboratories, Inc., Hercules, CA, U.S.A.).

Cell Culture We used the mouse colon carcinoma cell line (Colon26) and the mouse melanoma cell line (B16-F10) which cause lung metastasis. Colon26 expressing luciferase regularly (Colon26/Luc cells) was prepared in our laboratory. Briefly, to establish Colon26/Luc cells, Colon26 cells were transfected with plasmid DNA encoding luciferase reporter gene (pCMV-Luc) and selected by G418. The pCMV-Luc was constructed by subcloning the HindIII/XbaI firefly luciferase cDNA fragment from the pGL3-control vector (Promega, Madison, WI, U.S.A.) into the polylinker of the pcDNA vector (Invitrogen). B16-F10 cells was obtained from the Cell Resource Center for Biomedical Research (Tohoku University, Japan). Cells were maintained in RPMI 1640 supplemented with $10 \%$ FBS and antibiotics (culture medium) under a humidified atmosphere of $5 \% \mathrm{CO}_{2}$ in air at $37^{\circ} \mathrm{C}$.

In Vitro Gene Silencing Experiment Colon26/Luc or B16-F10 cells were plated on 24-well plates (Becton, Dickinson and Company, Franklin Lakes, NJ, U.S.A.) at a density of $1.0 \times 10^{4}$ cells $/$ well and cultivated in $500 \mu \mathrm{L}$ culture medium. In the silencing experiment, after $24 \mathrm{~h}$ pre-incubation, the medium was replaced with $500 \mu \mathrm{L}$ Opti-MEM I (transfection medium) and each complex containing $1 \mu \mathrm{g}$ siRNA was added to the cells and incubated for $2 \mathrm{~h}$. The mixed solution of solution of 5\% dextrose solution and DEPC-treated water, which was solvent of complexes, was added to the cells as a control (untreated cells). Then, the medium was replaced with culture medium and cells were cultured for a further $22 \mathrm{~h}$ at $37^{\circ} \mathrm{C}$. For the luciferase assay of Colon26/Luc cells, after $22 \mathrm{~h}$ incubation, the cells were washed with phosphate-buffered saline (PBS) and then lysed in $100 \mu \mathrm{L}$ lysis buffer ( $\mathrm{pH} 7.8$ and $0.2 \mathrm{M}$ Tris- $\mathrm{HCl}$ buffer containing $2 \mathrm{~mm}$ EDTA and $0.05 \%$ Triton $\mathrm{X}-100)$. Ten microliters of lysate samples were mixed with $50 \mu \mathrm{L}$ luciferase assay buffer (PicaGene; Toyo Ink Co., Ltd., Tokyo, Japan) and the light produced was immediately measured using a luminometer (Lumat LB 9507; EG \& G Berthold, Bad Wildbad, Germany). The protein content of lysate was determined by the Bradford assay (Bio-Rad Laboratories, Inc.) using BSA as a standard. Absorbance was measured using a microplate reader (Sunrise RC-R; Tecan Japan Co., Ltd., Kanagawa, Japan) at $595 \mathrm{~nm}$. Luciferase activity was recorded as relative light units (RLU) per mg protein and the results are shown as a percentage of untreated cells (control).

Cytotoxicity Cytotoxicity of various complexes on Colon26/Luc cells was measured using a commercially avail- 
able WST-1 cell proliferation reagent. WST-1 reagent was prepared (5 mM WST-1 and $0.2 \mathrm{~mm} 1$-methoxy PMS in PBS) and filtered through a $0.22 \mu \mathrm{m}$ filter (Millex-GP; Millipore Co., Bedford, MA, U.S.A.) just before the experiments. Colon26/ Luc cells were plated on 24-well plates or 96-well plates (Becton, Dickinson and Company) at a density of $1.0 \times 10^{4}$ or $5.0 \times 10^{3}$ cells/well in the culture medium. Each complex containing $1 \mu \mathrm{g}$ siRNA in $100 \mu \mathrm{L}$ Opti-MEM I was added to each well and incubated for $2 \mathrm{~h}$. The mixed solution of solution of 5\% dextrose solution and DEPC-treated water was added to the cells as a control (untreated cells). After incubation, the medium was replaced with $100 \mu \mathrm{L}$ culture medium and incubated for another $22 \mathrm{~h}$. The medium was replaced with $100 \mu \mathrm{L}$ culture medium, and then $10 \mu \mathrm{L}$ WST-1 reagent was added to each well. The cells were incubated for an additional $2 \mathrm{~h}$ at $37^{\circ} \mathrm{C}$, and absorbance was measured at a wavelength of $450 \mathrm{~nm}$ with a reference wavelength of $630 \mathrm{~nm}$, using a microplate reader. The results are shown as a percentage of untreated cells (control).

Cellular Uptake of Complex To visualize the uptake of the complexes, the cells were transfected by complexes constructed with Alexa Fluor-labeled siRNA, FITC-PEI, and $\gamma$-PGA. Fluorescence was observed with fluorescence microscopy (200× magnification; BZ-9000; KEYENCE, Osaka, Japan).

Animals Animal care and experimental procedures were performed in accordance with the Guidelines for Animal Experimentation of Nagasaki University with approval from the Institutional Animal Care and Use Committee. Male ddY mice (6 weeks old) were purchased from Japan SLC (Shizuoka, Japan). After shipping, mice were acclimatized to the environment for at least one day before experiments.

Agglutination Study Erythrocytes from mice were washed three times at $4^{\circ} \mathrm{C}$ by centrifugation at $5000 \mathrm{rpm}$ (Kubota 3500; Kubota, Tokyo, Japan) for $5 \mathrm{~min}$ and resuspended in PBS. A $2 \%(\mathrm{v} / \mathrm{v})$ stock suspension was prepared. Various complexes were added to the erythrocytes (complexes: stock suspension=1:1). The suspensions were incubated for $30 \mathrm{~min}$ at room temperature. Ten microliters of these suspensions were placed on a glass plate and agglutination was observed by microscopy (200× magnification).

Quantitative Real-Time Reverse Transcription Polymerase Chain Reaction (RT-PCR) Total RNA was isolated from B16-F10 cells using a GenElute Mammalian Total RNA Miniprep Kit (Sigma-Aldrich Co., LLC). The concentration of RNA was measured by NanoDrop ND-1000 spectrophotometer (Thermo Fisher Scientific Inc., Waltham, MA, U.S.A.). Reverse transcription of mRNA was carried out using the PrimeScript RT reagent Kit (TaKaRa Bio Inc., Shiga, Japan). Gene expression was assessed by real-time PCR using SYBR Premix Ex Taq II (TaKaRa Bio Inc.) and Stratagene Mx3000P (Agilent Technologies, Inc., Santa Clara, CA, U.S.A.). GAPDH expression was normalized to $\beta$-actin expression (GAPDH $/ \beta$ actin), and the results are shown as a percentage of untreated cells (control).

Statistical Analysis Statistical significance among groups was identified by Dunnett's pairwise multiple comparison test.

\section{RESULTS}

Physicochemical Properties and Electrophoresis Assay of siRNA/PEI Complex The particle size and $\zeta$-potential of
siRNA/PEI complexes are shown in Table 1.

The siRNA/PEI complexes, excepted siRNA/PEI4 complex, were approximately $73-102 \mathrm{~nm}$ in particle size and $45-52 \mathrm{mV}$ in $\zeta$-potential.

A gel retardation assay was employed to examine the release of siRNA from siRNA/PEI complexes (Fig. 1). Naked siRNA was detected as a band on the agarose gel. On the other hand, no band was detected in the lanes of siRNA/PEI complexes.

In Vitro Gene Silencing Effect of siRNA/PEI Complex Colon26/Luc cells were transfected with various complexes, and silencing effects were examined and are shown in Fig. 2A. The commercial vector, siRNA/Lipofectamine complex, showed a strong silencing effect of siRNA. The siRNA/PEI4 and siRNA/PEI8 complexes did not silence the luciferase activity of Colon26/Luc cells. The silencing effect of siRNA/PEI complexes was increased with an increase of PEI, and siRNA/ PEI complexes with a charge ratio greater than 16 showed significant luciferase knockdown. No silencing effect was confirmed in siRNA/PEI16 complexes using scramble siRNA (Fig. 2B). Also, siRNA/PEI16 complexes showed no cytotoxicity under this experimental condition (Fig. 2C).

Physicochemical Properties and Electrophoresis Assay of siRNA/PEI/ $\gamma$-PGA Complex In the preliminary experiment, the binary complex of siRNA with a high amount of PEI showed strong cytotoxicity. Therefore we developed a ternary complex including $\gamma$-PGA, which was reported to decrease the cytotoxicity of cationic complex without decreasing gene expression. ${ }^{27)}$ The particle size and $\zeta$-potential of siRNA/ PEI16/ $\gamma$-PGA complexes are shown in Table 2. The addition of $\gamma$-PGA to the binary complex (siRNA/PEI16 complex)

Table 1. Size and $\zeta$-Potential of siRNA/PEI Complexes

\begin{tabular}{lcc}
\hline \hline Complexes & Size $(\mathrm{nm})$ & $\zeta$-Potential $(\mathrm{mV})$ \\
\hline siRNA/PEI4 & $1388.0 \pm 451.7$ & $16.4 \pm 0.2$ \\
siRNA/PE8 & $102.2 \pm 32.8$ & $44.7 \pm 0.5$ \\
siRNA/PE12 & $80.8 \pm 23.0$ & $47.8 \pm 1.3$ \\
siRNA/PE16 & $94.1 \pm 47.0$ & $50.4 \pm 1.4$ \\
siRNA/PE20 & $87.3 \pm 16.5$ & $52.0 \pm 1.1$ \\
siRNA/PE24 & $73.2 \pm 7.9$ & $51.6 \pm 0.5$ \\
\hline
\end{tabular}

Each value represents the mean \pm S.D. $(n=3)$.

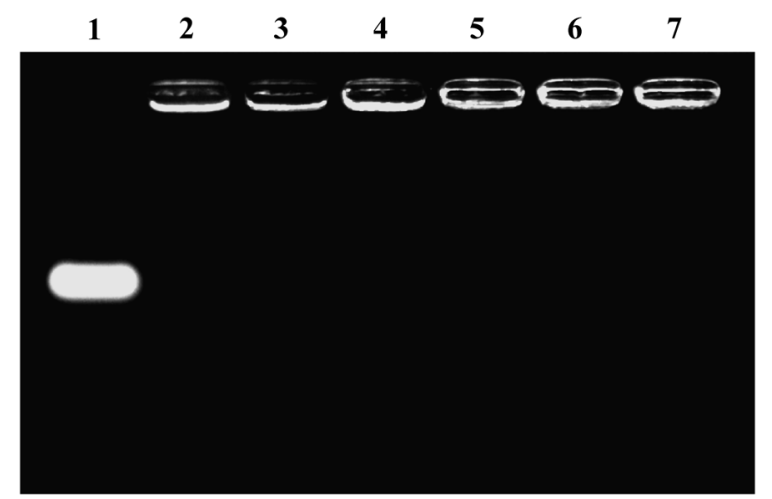

Fig. 1. Gel Retardation Assay of siRNA/PEI Complexes

The siRNA/PEI complexes were loaded onto agarose gel, and electrophoresis was carried out. Naked siRNA was run in lane 1. The siRNA was visualized with ethidium bromide staining. siRNA/PEI4 complex (lane 2), siRNA/PEI8 complex (lane 3), siRNA/PEI12 complex (lane 4), siRNA/PEI16 complex (lane 5), siRNA/ PEI20 complex (lane 6), siRNA/PEI24 complex (lane 7). 
(A)

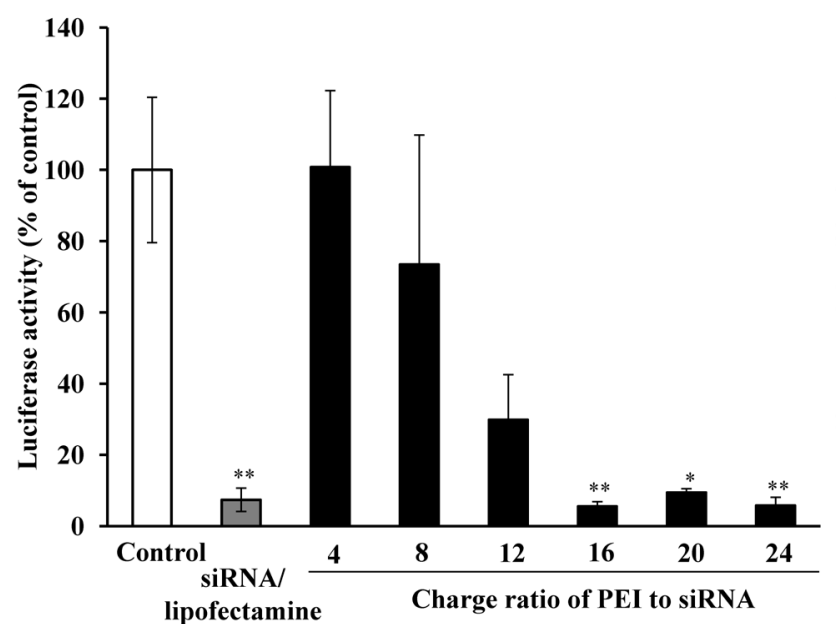

(B)

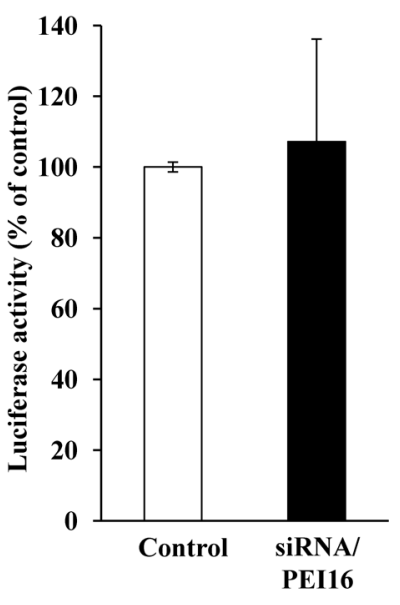

(C)

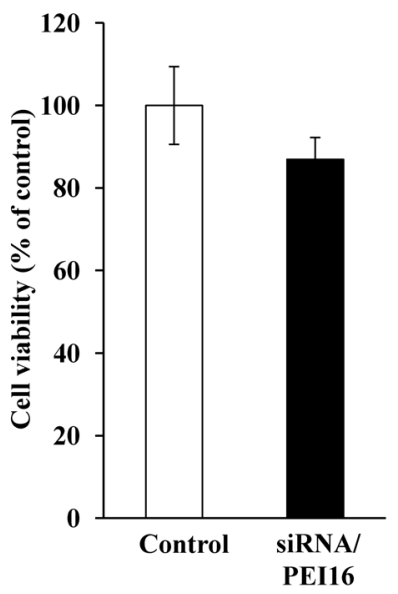

Fig. 2. In Vitro Gene Silencing of siRNA/PEI Complexes Using Firefly Luciferase siRNA (A) and Scramble siRNA (B) and Cytotoxicity of siRNA/ PEI16 Complexes in Colon26/Luc Cells (C)

Colon26/Luc cells were incubated with siRNA/Lipofectamine complex and siRNA/PEI complexes for $2 \mathrm{~h}$. After $22 \mathrm{~h}$, luciferase activity of cells was analyzed, normalized with the protein concentration and expressed as a percentage of luminescence intensity compared to the control. Cell viability after treatment with complexes was determined by WST-1 assay. Each bar represents the mean \pm S.E. $(n=3)$. ${ }^{*} p<0.05, * * p<0.01 v s$. control.

Table 2. Size and $\zeta$-Potential of siRNA/PEI/ $\gamma$-PGA Complexes

\begin{tabular}{lcc}
\hline \hline Complexes & Size $(\mathrm{nm})$ & $\zeta$-Potential $(\mathrm{mV})$ \\
\hline siRNA/PEI16/ $\gamma$-PGA4 & $90.9 \pm 16.1$ & $36.4 \pm 0.6$ \\
siRNA/PEI16/ $/$-PGA8 & $231.3 \pm 27.6$ & $-9.2 \pm 0.5$ \\
siRNA/PEI16/ $\gamma$-PGA12 & $107.6 \pm 1.8$ & $-26.5 \pm 0.6$ \\
siRNA/PEI16/ $\gamma$-PGA16 & $83.8 \pm 9.6$ & $-35.5 \pm 0.5$ \\
siRNA/PEI16/ $\gamma$-PGA20 & $85.1 \pm 65.2$ & $-46.4 \pm 1.9$ \\
\hline
\end{tabular}

Each value represents the mean \pm S.D. $(n=3)$.

decreased the $\zeta$-potential in a concentration-dependent manner. All ternary complexes (siRNA/PEI16/ $\gamma$-PGA complexes), except siRNA/PEI16/ $\gamma$-PGA8 complex, were approximately $41-108 \mathrm{~nm}$ in particle size with an anionic surface charge less than $-25 \mathrm{mV}$.

A gel retardation assay was employed to examine the release of siRNA from ternary complexes (Fig. 3). Naked siRNA was detected as a band on the agarose gel. Addition of a large amount of $\gamma$-PGA to the binary complex (siRNA/PEI16 complex) showed a slight release of siRNA, although ternary complexes including less than a charge ratio of 12 for $\gamma$-PGA to siRNA showed no band of naked siRNA.

Cytotoxicity of Binary Complex and Ternary Complex The cytotoxicity of binary complexes and ternary complexes was evaluated in Colon26/Luc cells using the WST-1 assay (Fig. 4). The siRNA/Lipofectamine complex and siRNA/ PEI16 complex, which had efficient silencing activity, showed significantly higher toxicity than the control. An increase of $\gamma$-PGA in ternary complexes decreased their cytotoxicity. The siRNA/PEI16/ $\gamma$-PGA12 complex did not affect the cell viability of colon26/Luc cells.

In Vitro Gene Silencing Effect of siRNA/PEI/ $\gamma$-PGA Complex All ternary complexes (siRNA/PEI/ $\gamma$-PGA complexes) were added and incubated with Colon26/Luc cells and silencing effects were determined (Fig. 5A). Strong suppression of luciferase expression in Colon26/Luc cells was observed in binary complexes (siRNA/Lipofectamine and

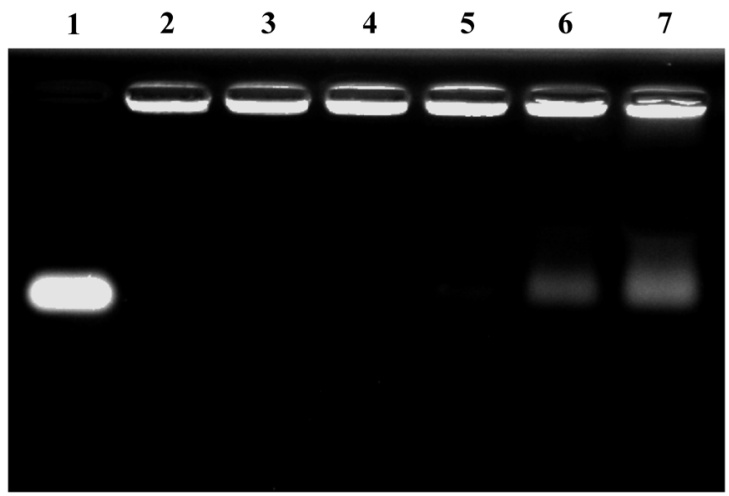

Fig. 3. Gel Retardation Assay of siRNA/PEI $/ \gamma$-PGA Complexes

The siRNA/PEI $/ \gamma$-PGA complexes were loaded onto agarose gel, and electrophoresis was carried out. Naked siRNA was run in lane 1 . The siRNA was visualized with ethidium bromide staining. siRNA/PEI16 complex (lane 2), siRNA/ PEI16/ $\gamma$-PGA4 complex (lane 3), siRNA/PEI16/ $\gamma$-PGA8 complex (lane 4), siRNA PEI16/ $\gamma$-PGA12 complex (lane 5), siRNA/PEI16/ $\gamma$-PGA16 complex (lane 6), siRNA/PEI16/ $\gamma$-PGA20 complex (lane 7 ).

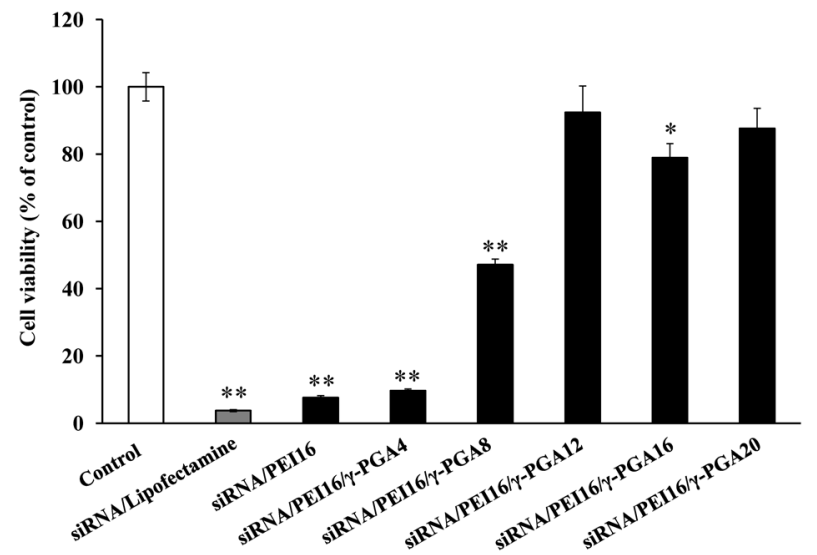

Fig. 4. Cytotoxicity of Complexes in Colon26/Luc Cells

Cell viability after treatment with complexes was determined by WST-1 assay Each bar represents the mean \pm S.E. $(n=8-16)$. ${ }^{*} p<0.05,{ }^{* *} p<0.01 v s$. control. 
siRNA/PEI16 complexes). Ternary complexes (siRNA/PEI16/ $\gamma$-PGA complexes) also showed a significant silencing effect. On the other hand, siRNA/PEI16/ $\gamma$-PGA12 complexes using scramble siRNA showed no silencing effect (Fig. 5B).

Cellular Uptake of Complex The cellular uptake of complex was visualized using Alexa Fluor 555-labeled siRNA and FITC-PEI, shown in Fig. 6. The binary complex (siRNA/ PEI16 complex) showed strong signals of the red fluorescence of Alexa Fluor 555-labeled siRNA and green fluorescence of FITC-labeled PEI inside the cells. Red and green fluorescence inside the cells were also observed in siRNA/PEI16/ $\gamma$-PGA12 complex. In the merged image, the complex was mostly observed as yellow signal in the cells, but red signal was also observed.

Agglutination Study The agglutination activities of the siRNA/PEI16/ $\gamma$-PGA12 complex were compared to that of the siRNA/PEI16 complex in erythrocytes and are shown in Fig. 7. The siRNA/PEI16 complex showed severe agglutination, although no agglutination was observed in the siRNA/ PEI16/ $\gamma$-PGA12 complex.
(A)

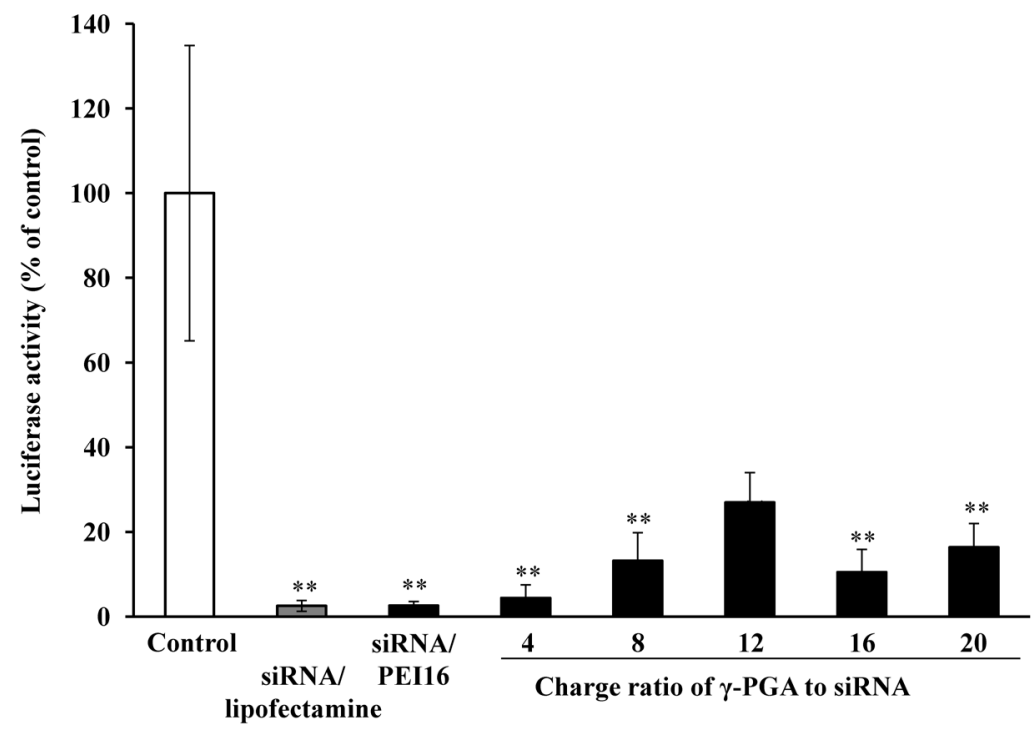

(B)

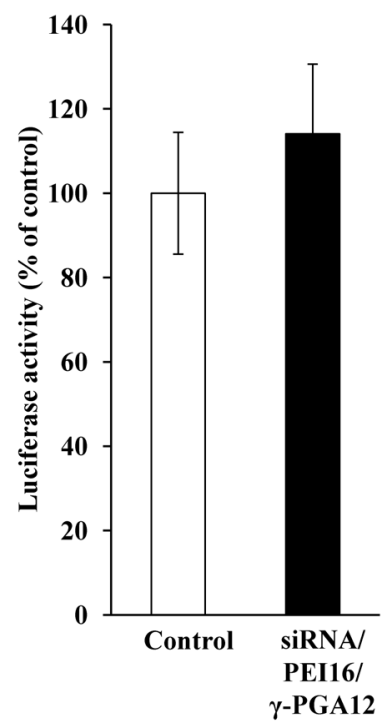

Fig. 5. In Vitro Gene Silencing of siRNA/PEI/ $\gamma$-PGA Complexes

Colon26/Luc cells were incubated with siRNA/Lipofectamine complex, siRNA/PEI complex, and siRNA/PEI/ $\gamma$-PGA complexes for $2 \mathrm{~h}$. After $22 \mathrm{~h}$, luciferase activity of cells was analyzed, normalized with the protein concentration and expressed as a percentage of luminescence intensity compared to the control. Each bar represents the mean \pm S.E. $(n=3) .{ }^{* *} p<0.01$ vs. control. (A): Firefly luciferase siRNA, (B): Scramble siRNA.
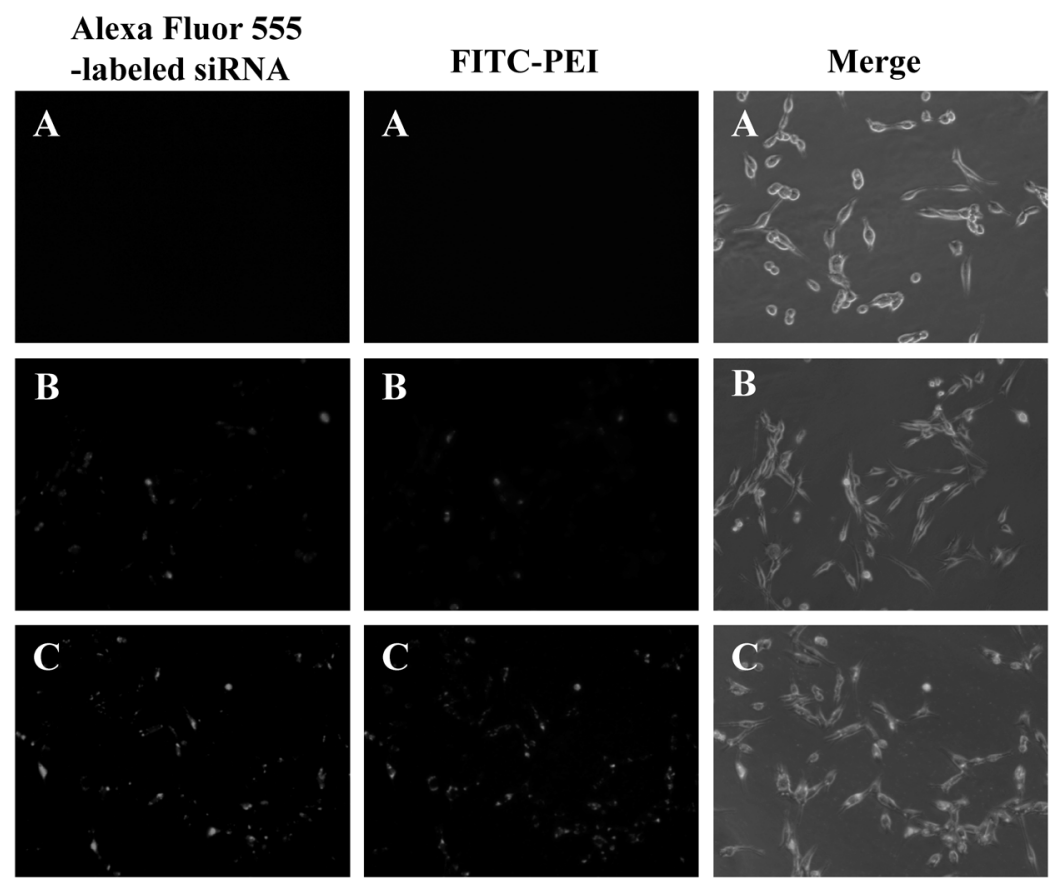

Fig. 6. Cellular Uptake of Complexes

Colon26/Luc cells were incubated with complexes containing Alexa Fluor 555-labeled siRNA and FITC-PEI, and fluorescent images were taken by fluorescence microscopy. (A): Control, (B): siRNA/PEI16 complex, (C): siRNA/PEI16/ $\gamma$-PGA12 complex. 

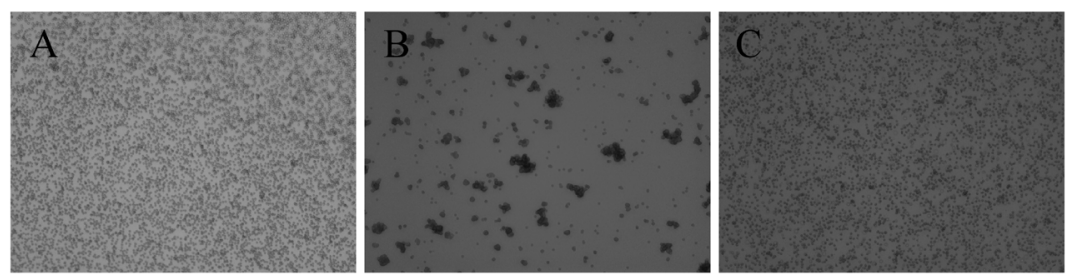

Fig. 7. Agglutination of Complexes with Erythrocytes

Each complex was added to erythrocytes, and agglutination was observed by microscopy (200× magnification). (A): Control, (B): siRNA/PEI16 complex, (C): siRNA/ PEI16/ $\gamma$-PGA12 complex.
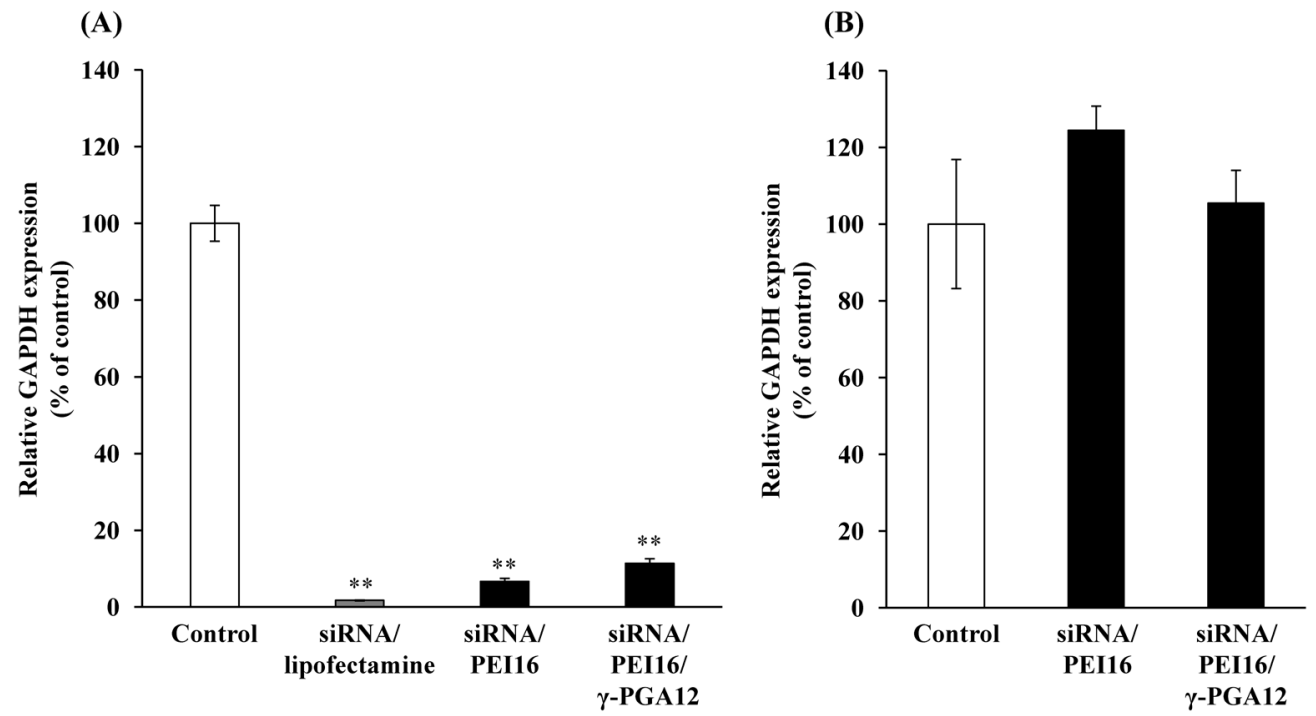

Fig. 8. GAPDH mRNA Knockdown of Complexes in B16-F10 Cells

B16-F10 cells were incubated with complexes for $2 \mathrm{~h}$. After $22 \mathrm{~h}$, GAPDH mRNA expression was measured using real-time RT-PCR, normalized to $\beta$-actin expression $(\mathrm{GAPDH} / \beta$-actin), and expressed as a percentage compared to the control. Each bar represents the mean $\pm \mathrm{S}$.E. $(n=3)$. $* * p<0.01 v s$. control. (A): GAPDH siRNA, (B): Scramble siRNA.

In Vitro GAPDH mRNA Knockdown To evaluate the silencing effect of complexes for endogenous proteins, mRNA of a housekeeping gene, GAPDH, was determined with real-time PCR in B16-F10 cells $24 \mathrm{~h}$ after incubation with complexes for $2 \mathrm{~h}$ (Fig. 8A). Significant reduction of GAPDH expression in B16F-10 cells was observed in binary complexes (siRNA/Lipofectamine and siRNA/PEI16 complexes). The ternary complex (siRNA/PEI16/ $\gamma$-PGA12 complex) also showed strong GAPDH mRNA knockdown. There were no knockdown effects in both binary complexes and ternary complexes using scramble siRNA (Fig. 8B).

\section{DISCUSSION}

siRNA formed an RNA-induced silencing complex (RISC) that consisted of an endoribonuclease. On formation of the RISC, siRNA strands direct the complex to the target complementary strand of RNA molecules, leading to destruction of the target RNA molecule in the cytoplasm. ${ }^{29)}$ siRNA technology has the potential to revolutionize the treatment of serious diseases by allowing selective silencing of oncogenic or therapeutic mRNAs and their corresponding proteins. The delivery of siRNA typically yields low levels of mRNA silencing due to barriers that include degradation by endo- and exonucleases, poor cellular uptake, and intracellular trafficking. ${ }^{30)}$ Polymer complexes have an advantage over lipid complexes in terms of stability in physiological media. ${ }^{31)}$ Polymer complexes called polyplexes can be defined as subcellular colloidal particles that entrap drug molecules, proteins, or nucleic acid. ${ }^{32)}$ Figure 2A shows the strong silencing effect of siRNA/PEI complexes on a mouse colon carcinoma cell line expressing luciferase regularly. An increase of the PEI charge ratio in the complexes increased the silencing effect. Also, the cell viability of siRNA/PEI16 complexes was almost equal to control (Fig. 2C). These results indicated that binary complexes did not induce cytotoxicity in this condition. The siRNA/PEI complexes at greater than charge ratio 12 of PEI to siRNA formed stable cationic particles of less than $100 \mathrm{~nm}$ in diameter and higher than $45 \mathrm{mV}$ in $\zeta$-potential (Table 1, Fig. 1). In the previous report, we demonstrated that plasmid DNA/PEI complex showed effective gene expression at greater than charge ratio $8 .{ }^{27)}$ Cationic agent-condensed plasmid DNA can exist in a variety of different morphologies depending upon the cationic condensing agent, such as spheres, toroids, and rods. ${ }^{33,34)}$ Electrostatic interactions between siRNA and cationic polymer increase the PEI amount for siRNA delivery. In contrast to plasmid DNA, siRNA cannot condense into particles of nanometric dimensions, being already a small subnanometric nucleic acid.

On the other hand, cytotoxicity and blood agglutination were induced by the cationic surface of the polyplexes. ${ }^{19,20)}$ The cationic complex was reported to bind to anionic proteoglycans on the cellular membrane, destabilizing the membrane and causing severe cytotoxicity. ${ }^{35-37)}$ The erythrocyte surface 
was also negatively charged to be agglutinated with cationic complexes. Agglutination of erythrocytes by positively charged complexes can result in the occlusion of capillaries in the lungs, which could account for the high toxicity. ${ }^{20,23)}$ In fact, strong cytotoxicity and blood agglutination were observed in the siRNA/Lipofectamine complex and siRNA/ PEI16 complex (Figs. 4, 7).

Recharging cationic complexes with an anionic compound was reported to be a promising method for overcoming these toxicities. ${ }^{25,26)}$ In the previous study, we successfully prepared various ternary complexes of plasmid DNA and PEI by the addition of anionic polymers such as polyadenylic acid, polyinosinic-polycytidylic acid, polyaspartic acid, polyglutamic acid, fucoidan, $\lambda$-carrageenan, xanthan gum, alginic acid, chondroitin sulfate, and hyaluronic acid. ${ }^{27,28)}$ All showed anionic surface charges with no cytotoxicity and agglutination. However, most showed no gene expression because of the lack of a strong interaction with cell and erythrocyte surfaces. Among them, we discovered high cellular uptake and high gene expression in the complexes of plasmid DNA and PEI with $\gamma$-PGA, chondroitin sulfate, or hyaluronic acid. The complex including $\gamma$-PGA showed the highest gene expression via a $\gamma$-PGA-specific receptor-mediated energy-dependent process. ${ }^{27)}$ Complexes including a plasmid encoding Plasmodium yoelii merozoite surface protein 1-C-terminus completely suppressed a lethal malaria challenge in a murine model by strong vaccination. ${ }^{38)}$

We prepared ternary complexes of siRNA with PEI (at charge ratio 16) by the addition of $\gamma$-PGA to reduce cytotoxicity with efficient delivery. As expected, the cytotoxicity of ternary complexes decreased with an increase of $\gamma$-PGA content, which decreased $\zeta$-potential of the complexes (Fig. 4, Table 2). siRNA/PEI16/ $\gamma$-PGA12 with an anionic surface charge showed no blood agglutination. The large addition of $\gamma$-PGA to the complexes enhanced the release of siRNA, suggesting destabilization of the complexes (Fig. 3).

As projected, a strong silencing effect, comparable to the siRNA/Lipofectamine complex, was observed in ternary complexes including $\gamma$-PGA, regardless of the anionic surface charge (Fig. 5). The high incorporation of ternary complexes into Colon26/Luc cells was also confirmed with fluorescence microcopy (Fig. 6). The number of intracellular siRNA of ternary complex may be observed larger than that of binary complex. However, the silencing effect of ternary complex showed lower than that of binary complex. The endosomal escape, siRNA release from complexes, and degradation of siRNA in the cells also have an impact on the silencing effects. We will examine the endosomal escape, siRNA release from complexes, and degradation of siRNA in the cells to explain the different gene silencing effects of various complexes in the future study.

Having achieved knockdown of an exogenously transfected gene, the ability of the complex to mediate knockdown of an endogenous housekeeping gene, GAPDH, was assessed in B16-F10 cells. The binary complexes (siRNA/Lipofectamine and siRNA/PEI16 complexes) showed strong GAPDH mRNA knockdown (Fig. 8A). The ternary complex (siRNA/PEI16/ $\gamma$-PGA12) with an anionic surface charge also exhibited a significant GAPDH mRNA knockdown effect (Fig. 8A).

Thus, we developed a ternary complex of siRNA with PEI by the addition of $\gamma$-PGA, showing a high silencing effect and no cytotoxicity, as a novel siRNA delivery system.

\section{REFERENCES}

1) Fire $\mathrm{A}, \mathrm{Xu} \mathrm{S}$, Montgomery MK, Kostas SA, Driver SE, Mello CC. Potent and specific genetic interference by double-stranded RNA in Caenorhabditis elegans. Nature, 391, 806-811 (1998).

2) Hammond SM, Bernstein E, Beach D, Hannon GJ. An RNAdirected nuclease mediates post-transcriptional gene silencing in Drosophila cells. Nature, 404, 293-296 (2000).

3) Bernstein E, Caudy AA, Hammond SM, Hannon GJ. Role for a bidentate ribonuclease in the initiation step of RNA interference. Nature, 409, 363-366 (2001).

4) Zamore PD, Tuschl T, Sharp PA, Bartel DP. RNAi: double-stranded RNA directs the ATP-dependent cleavage of mRNA at 21 to 23 nucleotide intervals. Cell, 101, 25-33 (2000).

5) Jones L, Hamilton AJ, Voinnet O, Thomas CL, Maule AJ, Baulcombe DC. RNA-DNA interactions and DNA methylation in posttranscriptional gene silencing. Plant Cell, 11, 2291-2301 (1999).

6) Smart N, Scambler PJ, Riley PR. A rapid and sensitive assay for quantification of siRNA efficiency and specificity. Biol. Proced. Online, 7, 1-7 (2005).

7) Tebes SJ, Kruk PA. The genesis of RNA interference, its potential clinical applications, and implications in gynecologic cancer. Gynecol. Oncol., 99, 736-741 (2005).

8) Reynolds A, Leake D, Boese Q, Scaringe S, Marshall WS, Khvorova A. Rational siRNA design for RNA interference. Nat. Biotechnol., 22, 326-330 (2004).

9) Tiemann K, Rossi JJ. RNAi-based therapeutics-current status, challenges and prospects. EMBO Mol. Med., 1, 142-151 (2009).

10) Kirchhoff F. Silencing HIV-1 in vivo. Cell, 134, 566-568 (2008).

11) Chien PY, Wang J, Carbonaro D, Lei S, Miller B, Sheikh S, Ali SM, Ahmad MU, Ahmad I. Novel cationic cardiolipin analoguebased liposome for efficient DNA and small interfering RNA delivery in vitro and in vivo. Cancer Gene Ther., 12, 321-328 (2005).

12) Basha G, Novobrantseva TI, Rosin N, Tam YY, Hafez IM, Wong MK, Sugo T, Ruda VM, Qin J, Klebanov B, Ciufolini M, Akinc A, Tam YK, Hope MJ, Cullis PR. Influence of cationic lipid composition on gene silencing properties of lipid nanoparticle formulations of siRNA in antigen-presenting cells. Mol. Ther., 19, 2186-2200 (2011).

13) Semple SC, Akinc A, Chen J, Sandhu AP, Mui BL, Cho CK, Sah DW, Stebbing D, Crosley EJ, Yaworski E, Hafez IM, Dorkin JR, Qin J, Lam K, Rajeev KG, Wong KF, Jeffs LB, Nechev L, Eisenhardt ML, Jayaraman M, Kazem M, Maier MA, Srinivasulu M, Weinstein MJ, Chen Q, Alvarez R, Barros SA, De S, Klimuk SK, Borland T, Kosovrasti V, Cantley WL, Tam YK, Manoharan M, Ciufolini MA, Tracy MA, de Fougerolles A, MacLachlan I, Cullis PR, Madden TD, Hope MJ. Rational design of cationic lipids for siRNA delivery. Nat. Biotechnol., 28, 172-176 (2010).

14) Huang YH, Bao Y, Peng W, Goldberg M, Love K, Bumcrot DA, Cole G, Langer R, Anderson DG, Sawicki JA. Claudin-3 gene silencing with siRNA suppresses ovarian tumor growth and metastasis. Proc. Natl. Acad. Sci. U.S.A., 106, 3426-3430 (2009).

15) Leuschner F, Dutta P, Gorbatov R, Novobrantseva TI, Donahoe JS Courties G, Lee KM, Kim JI, Markmann JF, Marinelli B, Panizzi P, Lee WW, Iwamoto Y, Milstein S, Epstein-Barash H, Cantley W, Wong J, Cortez-Retamozo V, Newton A, Love K, Libby P, Pittet MJ, Swirski FK, Koteliansky V, Langer R, Weissleder R, Anderson DG, Nahrendorf M. Therapeutic siRNA silencing in inflammatory monocytes in mice. Nat. Biotechnol., 29, 1005-1010 (2011).

16) Kodama Y, Harauchi S, Kawanabe S, Ichikawa N, Nakagawa H, Muro T, Higuchi N, Nakamura T, Kitahara T, Sasaki H. Safe and effective delivery of small interfering RNA with polymer- and liposomes-based complexes. Biol. Pharm. Bull., 36, 995-1001 (2013).

17) Kabanov AV. Taking polycation gene delivery systems from in vitro 
to in vivo. Pharm. Sci. Technol. Today, 2, 365-372 (1999).

18) Godbey WT, Wu KK, Mikos AG. Poly(ethylenimine) and its role in gene delivery. J. Control. Release, 60, 149-160 (1999).

19) Dekie L, Toncheva V, Dubruel P, Schacht EH, Barrett L, Seymour LW. Poly-L-glutamic acid derivatives as vectors for gene therapy. $J$. Control. Release, 65, 187-202 (2000).

20) Kircheis R, Wightman L, Schreiber A, Robitza B, Rössler V, Kursa M, Wagner E. Polyethylenimine/DNA complexes shielded by transferrin target gene expression to tumors after systemic application. Gene Ther., 8, 28-40 (2001).

21) Oskuee RK, Philipp A, Dehshahri A, Wagner E, Ramezani M. The impact of carboxyalkylation of branched polyethylenimine on effectiveness in small interfering RNA delivery. J. Gene Med., 12, 729-738 (2010)

22) Fischer D, Bieber T, Li Y, Elsässer HP, Kissel T. A novel non-viral vector for DNA delivery based on low molecular weight, branched polyethylenimine: effect of molecular weight on transfection efficiency and cytotoxicity. Pharm. Res., 16, 1273-1279 (1999).

23) Ogris M, Brunner S, Schüller S, Kircheis R, Wagner E. PEGylated DNA/transferrin-PEI complexes: reduced interaction with blood components, extended circulation in blood and potential for systemic gene delivery. Gene Ther., 6, 595-605 (1999).

24) Ogris M, Wagner E. Targeting tumors with non-viral gene delivery systems. Drug Discov. Today, 7, 479-485 (2002).

25) Trubetskoy VS, Wong SC, Subbotin V, Budker VG, Loomis A, Hagstrom JE, Wolff JA. Recharging cationic DNA complexes with highly charged polyanions for in vitro and in vivo gene delivery. Gene Ther., 10, 261-271 (2003).

26) Ito T, Iida-Tanaka N, Koyama Y. Efficient in vivo gene transfection by stable DNA/PEI complexes coated by hyaluronic acid. J. Drug Target., 16, 276-281 (2008).

27) Kurosaki T, Kitahara T, Fumoto S, Nishida K, Nakamura J, Niidome T, Kodama Y, Nakagawa H, To H, Sasaki H. Ternary complexes of pDNA, polyethylenimine, and gamma-polyglutamic acid for gene delivery systems. Biomaterials, 30, 2846-2853 (2009).

28) Kurosaki T, Kitahara T, Kawakami S, Nishida K, Nakamura J, Teshima M, Nakagawa H, Kodama Y, To H, Sasaki H. The development of a gene vector electrostatically assembled with a polysaccharide capsule. Biomaterials, 30, 4427-4434 (2009).

29) Leng Q, Woodle MC, Lu PY, Mixson AJ. Advances in systemic siRNA delivery. Drugs Future, 34, 721 (2009).

30) Shim MS, Kwon YJ. Efficient and targeted delivery of siRNA in vivo. FEBS J., 277, 4814-4827 (2010).

31) Zhang S, Zhao B, Jiang H, Wang B, Ma B. Cationic lipids and polymers mediated vectors for delivery of siRNA. J. Control. Release, 123, 1-10 (2007).

32) Hans ML, Lowman AM. Biodegradable nanoparticles for drug delivery and targeting. Curr. Opin. Solid State Mater. Sci., 6, 319-327 (2002).

33) Hansma HG, Golan R, Hsieh W, Lollo CP, Mullen-Ley P, Kwoh D. DNA condensation for gene therapy as monitored by atomic force microscopy. Nucleic Acids Res., 26, 2481-2487 (1998).

34) Golan R, Pietrasanta LI, Hsieh W, Hansma HG. DNA toroids: stages in condensation. Biochemistry, 38, 14069-14076 (1999).

35) Lv H, Zhang S, Wang B, Cui S, Yan J. Toxicity of cationic lipids and cationic polymers in gene delivery. J. Control. Release, 114, 100-109 (2006).

36) Moghimi SM, Symonds P, Murray JC, Hunter AC, Debska G, Szewczyk A. A two-stage poly(ethylenimine)-mediated cytotoxicity: implications for gene transfer/therapy. Mol. Ther., 11, 990-995 (2005).

37) Demeneix B, Behr JP. Polyethylenimine (PEI). Adv. Genet., 53, 217-230 (2005)

38) Cherif MS, Shuaibu MN, Kurosaki T, Helegbe GK, Kikuchi M, Yanagi T, Tsuboi T, Sasaki H, Hirayama K. Immunogenicity of novel nanoparticle-coated MSP-1 C-terminus malaria DNA vaccine using different routes of administration. Vaccine, 29, 9038-9050 (2011). 